Rev. Saúde Pública, 28 (2): 153-66, 1994

\title{
Evaluación económica y toma de decisiones en salud ambiental
}

\author{
Economic evaluation and decision-making in environmental health
}

\author{
Cristina Gutiérrez Zúñiga*, Patricia Hernández Peña**, Claudia Ortiz Guerrero**, Rocio Zurutuza \\ Fernández ${ }^{\star \star *}$, Carlos Santos-Burgoa* ${ }^{\star \star \star}$
}

\begin{abstract}
GUTTÉRREZ-ZUUNIGA, C. et al. Evaluación económica y toma de decisiones en salud ambiental. Rev. Saúde Pública, 28: 153-66, 1994. Ante el creciente deterioro ambiental y sus posibles consecuencias en la salud de la población resulta una prioridad indiscutible el diseño e instrumentación de políticas que controlen las actividades económicas bajo el criterio de protección a la salud humana y al ambiente mismo. Es preciso que dichas políticas consideren la factibilidad económica de las alternativas de protección existentes. Sin embargo, por la cantidad de intereses dentro del área ambiental, otros factores como el social y el político deben también ser considerados. La evaluación económica ha sido vista como un promisorio fundamento para la toma de decisiones en esta materia. Los autores analizan la capacidad de esta herramienta para organizar en forma sistemática y comparable los costos y los beneficios de alternativas para la solución de problemas ambientales. Se resumen las principales caracteristicas de los estudios de costo-beneficio y costo-efectividad, las formas de evaluación económica del ambiente, y las particularidades de esta área para la aplicación de dichas técnicas de análisis. Se señalan los limites encontrados en estas herramientas para cuantificar los costos no monetarios de los riesgos ambientales y de los consecuentes daños a la salud, tales como el dolor, el sufrimiento ó la incapacidad de personas económicamente inactivas, constituyendo estos aspectos el reto metodológico de la evaluación económica en el área. Se reflexiona sobre la importancia de ampliar los insumos informativos para la toma de decisiones en materia de salud ambiental en aspectos como la distribución de los costos y los beneficios entre los distintos grupos sociales. Por último se señala tanto la creciente politización del tema ambiental, como la posibilidad técnica de manipulación de estas herramientas de análisis. Partiendo de estos elementos se señala la necesidad, por parte de los evaluadores, de ser conscientes sobre las implicaciones politicas de sus estudios, así como de la importancia de su vinculación con los tomadores de decisión con el objetivo de lograr pertinencia en el ejercicio académico.
\end{abstract}

Descriptores: Analisis de costo-beneficio. Toma de decisiones. Salud ambiental, economia.

\section{1 - Introducción}

En los últimos años la dinámica de deterioro ambiental ha generado incertidumbre en la gran mayoría de los países. No existe aún una clara identificación de los peligros que implica ni de los procedimientos para enfrentarlos. Hay un desconocimiento considerable sobre los efectos en la salud que puedan producirse debido a este deterioro en el mediano y largo plazos. Las tecnologías

\footnotetext{
- El Colégio de Jalisco y Departamento de Economía de la Salud, Escuela de Salud Publica de México (ESP)

- Departamento de Economía de la Salud, Escuela de Salud Publica de México.

*.* Escuela de Salud Publica de México.

Separatas/Reprints:C. Gutiénez-Zúñiga - El Colegio de Jalisco Calle 5 de Mayo, 209 Zapopan - Jalisco - México
}

disponibles para modificar los procesos productivos en beneficio del ambiente y de la salud humana están creándose y enfrentan desde su diseño el reto de la eficacia y de la factibilidad económica. Ante tal situación, la formulación e instrumentación de políticas que normen las actividades económicas a fin de garantizar la protección del ambiente y de la salud constituye una prioridad de dificil discusión. Las evaluaciones económicas y en particular los estudios de costo-efectividad y costo-beneficio constituyen un insumo básico para fundamentar la toma de decisiones en esta materia ya que se encuentran orientadas hacia la identificación de las opciones que garanticen protección y sean económicamente viables. Sin embargo debido a la diversidad de intereses inmersos en esta área, las políticas de 
protección ambiental deben tener fundamento no sólo en los aspectos propiamente económicos, sino considerar también los factores políticos y sociales afectables como un insumo informativo imprescindible para su definición y adopción.

México no está al margen de esta preocupación. Actualmente se desarrolla un esfuerzo a nivel nacional para asegurar la protección del ambiente y el bicnestar de la sociedad. Esta intención está legalizada en la Lcy Federal de Metrología y Normalización ${ }^{1 / 4}$ en la que se explicita la necesidad de que todo proceso productivo y comercial cumpla con lineamientos que aseguren el beneficio de la población. A través de la elaboración de evaluciones económicas se espera apoyar la toma de decisiones en esta materia en favor de aquella alternativa o mecanismo que permita alcanzar el objetivo deseado con el mayor beneficio neto. ${ }^{14}$ En esta etapa, en la que los procesos de producción y de comercio así como la regulamentación hasta ahora vigente se encuentran en escrutinio bajo el criterio de la protección a la salud ambiental, es preciso preguntarse: iEs la evaluación económica una respuesta a la necesidad de información objetiva y comparable para la toma de decisiones en la materia? ¿Cuáles son sus alcances y sus límites?

Podemos decir que no existe hasta ahora una experiencia tecnológica y científica amplia en México que fundamente la toma de decisiones partiendo de las evaluaciones económicas. En particular, la experiencia extranjera en este sentido permite prevecr una serie de limitantes que deben ser consideradas en su oportunidad para obtener el máximo beneficio de la evaluación económica en cualquiera de sus formas. El éxilo de la nueva ley puede ser mayor si se toma provecho de la experiencia mencionada.

El presente trabajo tiene por objetivo analizar la capacidad de la evaluación económica para la toma de decisiones como alternativa para solucionar los problemas ambientales.

\section{2 - ¿Que es una Evaluación Económica?}

La evaluación cconómica es el análisis cuantitativo de los costos y resultados de la inversión en proyectos y programas alternativos ${ }^{15}$. Su linalidad es identificar la mejor utilización de los recursos a través de la estimación y comparación de costos y consecuencias de las opciones existentes para la consecución del objetivo de un proyecto o programa. Incluye también el análisis del costo de no emprencler ninguna acción frente a un problema planteado. Originalmente usado por los Cucrpos Armados de Ingenieros para juzgar la descabilidad de proyectos alternativos sobre recursos acuiferos, se ha convertido paulatinamente en un instrumento de análisis económico ampliamente utilizado tanto en la evaluación de proyectos de inversión en una empresa privada, como en el plano gubernamental a fin de valorar la conveniencia de cliversas posibilidades de intervención en bencficio dc la población, por ejemplo en el área de la salud ${ }^{10}$. Para cllo se han ido incorporando conocimientos interdisciplinarios según las necesidades de cada campo de aplicación, adoptando y creando metodologías que posibilitan la valoración cuantitativa de diversos aspectos anteriormente concebidos como no susceptibles de medición.

Dos métodos de evaluación económica frecuentemente utilizados son el análisis de costo-beneficio ( $A C B$ ) y el análisis de costo-efcctividad ( $\Lambda$ CE). El primero responde a la pregunta: ¿Cuál de las alternativas planteadas $A$ ó $B$ oliece un mayor beneficio neto, es decir un mayor bencficio una vez descontados sus gastos respectivos? El segundo respondería a la pregunta: ¿Cuál de la opciones A ó B alcanza el mismo objetivo por cl menor costo? o bien icuál de las opciones A ó B es más efectiva por un mismo costo?

La evaluación económica incluye tres pasos: $1^{\circ}$ identificación de costos y consecuencias de las alternativas a evaluar, en un lugar y un tiempo limitados; $2^{\circ}$ su valoración económica; y $3^{\circ}$ comparación de costos o de costos y consecuencias para la toma de decisiones. 
La realización de este tipo de análisis requiere por una parte, que exista una relación inequívoca entre la alternativa de acción planteada y el objetivo propuesto; además, deben considerarse hasta donde sea posible, la totalidad de los valores afectados positiva o negalivamente ${ }^{31}$; por otra, dado que estos valores se dan a lo largo de un período de tiempo definido, deben ajustarse los costos y consecuencias esperados descontando su valor futuro a valor presente neto.

\subsection{La valoración de costos y consecuencias}

Se define como costo el conjunto de los recursos absorbidos para la realización de una acción. Estos costos pueden ser directos e indirectos: los primeros son aquellos específicamente involucrados cn la realización de la acción y los segundos son los que se incurre en forma indirecta. En cl caso de una evaluación de alternativas para combatir una enfermedad específica, los costos directos son las erogaciones requeridas para el desarrollo del proyecto de salud, tales como compra de insumos (i.e. medicamentos), infraestructura de atención a la salud, etc. Estos bienes pueden ser valorados económicamente en forma inmediata ya que poseen un precio en $\mathrm{cl}$ mercado. Los indirectos se refieren a los costos en los que incurre el individuo por la utilización de su tiempo. En el caso de la evaluación de una enfermedad, el individuo (y frecuentemente un acompañante) desvía la utilización productiva de su tiempo para atender su salud, incluyendo en este costo el tiempo de espera o el tiempo de traslado a los servicios médicos.

Los beneficios por su parte son definidos como el valor económico de los efectos de la alternativa de acción considerada. Los beneficios se dividen a su vez en directos, inderectos e intangibles. En el caso considerado de abatimiento de una enfermedad, los primeros corresponden a la proporción de costos directos ahorrados en los servicios médicos por la inexistencia o reducción de la misma. Los segundos incluyen el ahorro creado al evitar el impacto de la enfermedad en la productividad o el ingreso debido a la ausencia laboral o reducción de la actividad. Este costo indirecto puede ser calculado partiendo del supuesto general de que el costo de oportunidad de tal enfermedad es el ingreso anual promedio según edad y sexo de quienes la padecen, o bien su colaboración per cápita al Producto Interno Bruto (PIB) del país. Generalmente este impacto económico es medido a través de la estimación de "días de vida saludables perdidos " y su correspondiente valor monetario en términos de ingreso o producto potencialmente generable por individuo. Esta medición combina información obtenida de la tasa de morbilidad, letalidad, extensión y cluración de la incapacidad, funcionando así como medida estandarizada para comparar diversas enfermedades ${ }^{7}$. En el caso de las incapacidades severas o bien de las enfermedades letales, la unidad utilizada son los "años de vida perdidos", los cuales estiman la diferencia entre expectativa de vida del individuo y su edad al adquirir la incapacidad o la edad de muerte 9 . Para obtener el equivalente monetario de esta pérdida, suele descontarse de la estimación a aquellos menores de 20 años de edad y mayores de 70 , por estar fuera de la Población Económicamente Activa (PEA). Como ejemplo diremos que los beneficios dircctos $\mathrm{e}$ indirectos del abatimiento de las enfermedades ocupacionales en los Estados Unidos son estimados en US $\$ 6,000$ millones anuales. ${ }^{6}$

Un método particularmente aplicado a enfermedades que crean condiciones crónicas es la llamada "años de vida ajustados por calidad" o QALYs (Quality Adjusted Life Years). Esta unidad ajusta los años de vida ganados gracias a una intervención por una medicla de la calidad de vida de la nueva condición limitada en la que sobrevive el individuo. Esta medida nos introduce al tercer tipo de beneficios considerados en una evaluación económica: los llamados beneficios intangibles. Estos beneficios están constituidos por el ahorro del dolor, la reducción de incomodidades y repercusiones de la enfermedad en el bienestar del individuo y de su familia. Debido a la inexistencia de un valor de mercado para 
dichos beneficios, así como a su caráter subjetivo, han significado un reto importante para el desarrollo metodológico de la evaluación económica. La técnica más usual para monetarizar su valor es la llamada "disponibilidad para pagar" (WTP, Willingness To Pay), que consiste en preguntar directamente a los individuos la cantidad de recursos monetarios (o proporción de su ingreso mensual) que estarían dispuestos a pagar por ese bien intangible. ${ }^{27}$ Otra técnica utilizada para la valoración de bienes sin un precio de mercado es la denominada "precios sombra".

\subsection{Análisis de Costo-Beneficio y de Costo-Efectividad}

El ACB al permitir conocer el bencficio neto de cada alternativa analizada para la consecución de un objetivo o programa, of rece un criterio objetivo para la comparación de opciones. Por cllo ha sido utilizado en cl área de salud. Los requisitos específicos para un $A C B$ consisten por una parte, en que los bencficios y los costos deben ser cuantificados en términos monetarios, y por otra, que todas las alternativas de solución factibles deben ser consideradas, así como el amplio espectro de costos y beneficios implicados en ellas.

El cumplimiento del primer requisito implica un mayor esfuerzo por parte del evaluador con respecto a otras técnicas de evaluación. Pero a su vez, al ponderar tanto costos como beneficios en forma amplia, sus resultados tienen una mayor capacidad de impacto en la toma de decisiones.

Por su parte la técnica de ACE permite evaluar programas alternativos destinados a lograr una misma meta examinando diversas alternativas relacionadas con magnitud, alcance y composición de un programa. Su finalidad es investigar la mejor manera de alcanzar un objetivo a través de comparar costos y efectos, evaluando el número posible de intervenciones para alcanzar el objetivo al menor costo, o bien, dado un presupuesto, la alternativa de acción que maximiza la efectividad del gasto.
El $\Lambda \mathrm{CE}$ tiene mayor flexibilidad que el $\mathrm{ACB}$ pucs no está limitado a cuantificar todos los efectos en valores monetarios: los resultados son expresados en términos ya sea de costo por unidad de producto, o bicn como el efecto obtenido por unidad monetaria gastada, por ejemplo costo monetario por muerte evitada.

Existe controversia acerca de la amplitud de costos y efectos de cada alternativa que deben ser medidos, ya que ésta puede ser considerable e incluso no referida estrictamente al objetivo en cuestión. En ese caso debe señalarse separadamente que la alternaliva está siendo reducida al objetivo propuesto para fines de comparabilidad con las otras, y debe indicarse los beneficios y costos que para la consecución de otros objetivos endría. 31

La obtención de beneficios netos o de razones de costo-efectividad comparables sistematizan y facilitan la elección de alternativas para enfrentar un problema. ${ }^{26}$

\section{3 - La Aplicación del ACB y ACE al Area Ambiental}

La aplicación de la evaluación económica al campo de la salud ambiental amerita de diversas adaptaciones a las características de esta área, empezando por la valoración económica del ambiente. Antes de disculir este punto es preciso distinguir dos ámbitos de aplicación tradicional de la evaluación económica en este campo: el microeconómico, y el macrocconómico. En el primero se busca clegir una altcrnativa de acción en función de la reducción de la contaminación que considere tanto la conveniencia empresarial en la inversión en tecnología ecológica, como el impacto en los costos y precios y su efecto en la demanda del producto generado. Sin embargo estas mediclas pueden tener un impacto en otros niveles más generales de la economía como son el empleo, la inversión, el PIB y la atención de las demandas sociales crecientes en la materia. ${ }^{19}$ Estos efectos son considerados en la evaluación aplicada al ámbito macrocconómico, sobre el que inciden 
las decisiones gubernamentales en materia de política económica, así como las políticas específicas enfocadas a la salud pública y al bienestar de la población. En este nivel cuya complejidad no permite una aplicación autómatica de categorías microeconómicas, centraremos nuestra atención.

\subsection{Las dimensiones económicas del ambiente}

La valoración económica del ambiente más tradicional se basa en que el suelo, el agua, la flora, la fauna y el aire son en su conjunto recursos naturales utilizados como insumos para los procesos productivos. Es a partir de ahí que poseen un precio en cl mercado, y que han podido ser valorados en forma directa como costos o como bencficios en las evaluaciones económicas. Reflexiones más recientes ponen en evidencia que la posibilidad de su agotamiento, deterioro o alteración plantea límites a la sustentabilidad del desarrollo económico. Incluso se ha llegado a plantear que el valor económico de los daños ambientales ya sean reversibles o irreversibles, comparados con el crecimiento económico gencrado, arroja como resultado un dudoso beneficio neto que cuestiona a los patrones actuales de desarrollo $y$ de uso de recursos adoptados ${ }^{21}$. En un ejercicio piloto realizado por el Banco Mundial se estima que de deducirse el costo del agotamiento de recursos naturales, deforestación, uso de la tierra y degradación del ambiente, el Producto Nacional Neto de México ascendería a $87 \%$ del anualmente reportado. 29

Un segundo nivel de valoración económica del ambiente es el que se refiere al deterioro de éste y sus efectos en la salud humana. En efecto, en la medida en la que se documenta la relación contaminación-enfermedad, el deterioro ambiental cuenta con una segunda posibilidad de valoración económica a través de las técnicas de costeo del impacto de la enfermedad reseñadas en la sección anterior. Esta dimensión nos proveé de uno de los criterios más sólidos para el diseño de políticas ambientales. Recientemente se ha reportado que a través de un cálculo a grosso modo podrían estimarse en US\$ 1,100 millones los costos en salud asociables a la contaminación atmosférica por ozono, partículas suspendidas totales (PST) y plomo en el Área Metropolitana de la Cd. de México. ${ }^{12}$

Un tercer nivel se refiere al valor estético-recreativo del ambiente. Este nivel es el más reciente y propone la valoración económica del ambiente por sí mismo. Afirma la posibilidad de valorar el ambiente - lagos, bosques, parques, playas - en tanto contiene un valor recreativo y estético susceptible de monctarización. Un ejemplo de este valor es que estamos dispuestos a pagar una cuota de entrada a un parque. Otras reflexiones relevan cl valor de este uso recreativo para la salud mental y la calidad de vida, que ameritan medios ad-hoc para su medición económica.

A partir de la utilidad del ambiente en estas dimensiones, productiva, de salud y estético-recreativa, podemos valorar económicamente costos y beneficios de medidas alternativas para la prevención y el mancjo de la contaminación ambiental.

\subsection{Particularidades del campo de la salud ambiental para la realización de evaluaciones económicas.}

La realización de evaluaciones económicas de alternativas de manejo ambiental, deben considerar diversas particularidades del campo. Entre ellas hemos elegido 4 que consideramos las más relevantes:

a) presencia de externalidades: Para considerar la problemática de la contaminación ambiental bajo una perspectiva económica es preciso señalar que los sistemas de mercado han fallado en cuanto al mancjo efectivo de la degradación ambiental, significando con ello que el medio ambiente no ha sido usado en forma óptima. ${ }^{16}$ La mayoría cle las empresas y unidades domésticas no han absorbido el costo de daño causado por sus emisiones, considerando los recursos naturales como bienes públicos y ocasionando así una divergencia entre los costos privados y los costos sociales. 5 
Bajo este contexto, se fundamenta la necesidad de la intervención económica del Estado para internalizar los costos ambientales, es decir, para corregir esta divergencia entre los costos privados y costos sociales, ya sea mediante mecanismos propios del mercado, o bien mediante otras acciones normativas y coercitivas. Definir la forma de esta intervención es una de las finalidades básicas de la evaluación económica en ambiente. Sin embargo, en proceso mismo de la valoración de costos y benefìcios es preciso tomar en consideración estas imperfecciones del mercado a fin de valorar adecuadamente toda alternativa de acción ambiental en beneficio del conjunto de la población.

b) el caráter meritorio de la salud y el bienestar: La evaluación económica debe tomar en cuenta al analizar costos y consecuencias de acciones alternativas en pro de la salud ambiental, que la afectación de los niveles de salud y de bienestar de la población no puede recibir un tratamiento igual a otros bienes y servicios $^{3}$ ya que existe una consideración de carácter ético en la sociedad que establece ya sea el derecho ciudadano a dichos bienes, el derecho universal en el gozo de los mismos, o bien el derecho a una satisfacción minima de necesidades en lo que a estos bienes respecta; estas posturas abarcan también el derecho de las futuras generaciones. Las alternativas consideradas en una evaluación económica no pueden ser analizadas bajo la óptica exclusiva de su factibilidad económica,sino en principio deben concordar con el marco ético y juridico que la sociedad en cuestión propone.La sensibilidad del evaluador a este respecto resulta vital.

c) complejidad de la salud ambiental como campo de conocimiento: La identificación de alternativas que efectivamente alcancen el objetivo deseado de protección del ambiente, de la salud humana o de los recursos naturales no es obvia. Por el contrario, el conocimiento de las interrelaciones ecológicas que afectan estos tres aspectos se encuentra en expansión, posibilitando el planteamiento de alternativas cada vez más eficaces y eficientes de
protección.Para plantear una alternativa regulatoria que evite el daño a la salud producido por una substancia tóxica deben contarse con estudios tanto clínicos como epidemiológicos que determinen las fuentes de dicha substancia,sus posibilidades de control,sus medios de transporte,sus posibilidades de contacto con el hombre,así como las dosis respuesta que nos permitan identificar los umbrales en los que la substancia implica un riesgo por sí o bien en sinergia con otras o en otras condiciones. Además de estos estudios que toman en consideración a la población en general, debe identificarse si existen poblaciones particularmente expuestas (factores ocupacionales, por ejemplo) o particularmente sensibles (niños,mujeres embarazadas, pacientes bajo ciertas concliciones crónicas) a dichas substancias y estabelecer regulaciones específicas para dicha exposición, incluyendo en última instancia opciones de reparación del daño.Existen alternativas distintas a la opción de emisión cero de una substancia tóxica que pueden proteger en forma efectiva la salud humana, o que bien,dada una imposibilidad técnica del abatimiento absoluto del riesgo, estabelecen limites aceptables y factibles del mismo.

d) incertidumbre: Las tres particularidades anteriores contribuyen a que la evaluación económica se realice en condiciones de incertidumbre; a cllo añadiremos que en la medida en que la investigación clínica epidemiológica avance en esta área,los resultados de las evaluaciones económicas previas perderían su sustento: una decisión distinta a la alternativa de emisión cero de una substancia tóxica puede ser un ahorro en el corto plazo a la luz de la información entonces vigente, pero un costo invaluable en el futuro a la luz de nuevas investigaciones. Existe una considerable ignorancia sobre los efectos en la salud humana de substancias existentes en el ambiente.Cerca del $80 \%$ de químicos industriales en uso en los Estados Unidos no han pasado por pruebas de toxidad ${ }^{11}$. Este hecho no es extraño considerando la multiplicidad de nuevas substancias generadas en nuestro tiempo, así como el que sus efectos en la salud 
pueden presentarse en el largo plazo y dentro de ámbitos geográficos extendidos. Por otra parte las mediciones de costos y beneficios deben ajustarse a períodos de tiempo considerables, aún cuando ignoramos la valoración que las generaciones futuras asignen tanto a los costos como a los beneficios ${ }^{10}$. De esta manera, la valoración estará necesariamente basada en criterios actuales. Todos estos elementos en su conjunto no son considerados obstáculos a la realización de evaluaciones económicas en el campo ambiental, sino condiciones técnicas específicas a superar a través de la ayuda de herramientas especializadas, así como de la creatividad y profesionalismo de los evaluadores.

\section{4 - Limites de la Evaluación Económica al Campo de la Salud Ambiental}

\subsection{Las Disyuntivas en la Toma de Decisiones Ambientales}

La asignación de recursos en el campo de la salud ambiental plantea la necesidad de priorización entre una multiplicidad de fines específicos en competencia y una cantidad aún mayor de medios para lograrlos. Ante la tradición de no cubrir los costos por contaminación, los efectos actuales y potenciales en la salud representan un costo no incorporado en la toma de decisiones sobre el control y manejo ambiental.Por otra parte, los efectos de la contaminación que ya existe o que serán generados en forma inminente ameritan de una manejo específico que implica un costo cuya importancia y magnitud compite con otras prioridades de atención a la salud. Este es el marco que hace necesaria la evaluación económica del ambiente. Si embargo, la toma de decisiones en el área no siempre encuentra respuesta en una razón de efectividad o un beneficio neto comparado.Por ejemplo, ante las diversas alternativas para desechar los lodos de una planta de tratamiento de aguas residuales no contamos con una tabla de equivalencias entre miles de casos de afecciones respiratorias que implicaría una opción y unos cuantos casos de cáncer que implicaría otra, o entre la destrucción de un acuario natural ahora o la extinción de una especie marina dentro de 1000 años. 23

La respuesta ante estas disyuntivas planteadas por la necesidad de acciones que enfrenten globalmente el problema y la escasez de recursos no es obvia.Es ingenuo pensar que un $A C B$ o un $A C E$ aunque útiles pudieran o debieran darnos un marco de referencia ético completo para analizar preguntas sobre la asignación de recursos. ${ }^{24}$

Si las evaluaciones económicas no pueden responder a estas cuestiones éticas inherentes a un proceso de decisión, la pregunta es entonces si la cvaluación puede por lo menos proveer de la información necesaria y suficiente para posteriormetne arribar a una decisión óptima en la materia, reflejando la opción que como sociedad deseamos tomar en materia ambiental.Para ello es necesario revisar algunos de sus límites al ser aplicada al área ambiental.

\subsection{El ACB y el ACE como fuentes de información sobre el bienestar de la población}

\subsubsection{Definición de límites espacio temporales}

Los estudios ambientales plantean relaciones espacio-temporales muy amplias: una derrama de una substancia tóxica en un punto geográfico puede traducirse en daños a la salud o al equilibrio ecológico en un punto opuesto de la 'Tierra cinco años después. O bien la devastación de una área boscosa puede llevar a la desertificación y la incapacidad productiva de la zona en la siguiente generación .Estas relaciones globales pasan por encima de fronteras políticas planteando paradojas a los tomadores de decisiones de países involucrados en dicha relación: para un país puede resultar un imperativo económico emplear recursos para el saneamiento ambiental no propio, sino de vecino.*

Una cvaluación económica tiene entonces el reto de abarcar en la medida de lo posible

- Jacobo Finkelman - Seminário de Salud Ambiental, México, 1992. 
estos impactos al definir sus límites espacio temporales. Un límite de ticmpo inadecuado se traduce en un sesgo de la evaluación contra aquellas alternativas que como las preventivas pudieran tener un efecto a largo plazo. ${ }^{22}$ Lo mismo puede aplicarse a aquellas alternativas de acción que plantean beneficios más amplios que los establecidos en el objetivo del proyecto - programa.En algunos casos la amplitud de estas relaciones globales empieza a ser conocida.En la mayoria, aún no.Otras veces esta amplitud espacio temporal sobrepasa los intereses prácticos del evaluador o el tomador de decisiones, quien deja de considerar en la práctica además de los efectos desconocidos, los de largo plazo y los geográfica o políticamente ajenos. Otras veces esta amplitud es simplemente inabarcable,produciéndose por definición un sesgo sistemático en favor de los costos y consecuencias a corto y mediano plazo, así como de los que tienen lugar en los límites nacionales.

\subsubsection{Medición de costos y consecuencias}

La medición cuantitativa para fines de comparabilidad requerida por la evaluación económica obliga a recurrir frecuentemente a métodos de valoración indirecta en al área ambiental debido a la presencia de numerosos intangibles.Es inevitable el riesgo de una medición arbitraria o sesgada en contra de aquellos bienes no fácilmente cuantificables. ${ }^{20}$ Aquellos aspectos que pueden ser más fácilmente medidos, se refieren clásicamente a los costos por ejemplo, el manejo de una enfermedad desde la perspectiva de los servicios de salud, o la pérdida del PIB por una disminución en la productividad, o el costo que representa para una industria instalar una tecnología anticontaminante o un sistema de reciclamiento....Menor atención ha recibido la medición del impacto que produce la enfermedad de individuos fuera del mercado laboral, como los niños o las amas de casa, el impacto en la distribuición del gasto doméstico por enfermedad, la disminuición en la calidad de vida del individuo por el dolor de una enfermedad, la pérdida de tiempo libre y de un espacio de recreación. ${ }^{27}$ Para los Estados Unidos se ha estimado que el costo de una enfermedad en uso de servicios médicos y pérdidas en la productividad constituye la mitad de los costos intangibles medidos a través de la técnica WTP. ${ }^{2}$ Medir en forma realista estos aspectos constituye el reto metodológico actual del $\Lambda C B$ y $A C E$. Comparativamente a los costos monetarios de las entidades públicas o de los actores económicos,los costos de adaptación del individuo y del espacio doméstico a la enfermedad y al deterioro ambiental son todavía inexactos. Esta incxactitud se vuelve protagónica cuando se pretende que una evaluación económica provea información comparable sobre el impacto de medidas diversas en el bienestar de la población. ${ }^{13}$

\subsubsection{Distribuición de costos y beneficios}

El análisis de costo beneficio está basado en la idea del mejoramiento potencial de Pareto,csto es, es aceptable una alternativa de acción en la que cl bencficio de los ganadores es suficiente como para compensar a los perdedores y aún exceder dicha compensación. Esta posición asume que tanto los costos como las ganancias se encuentran distribuidas aleatoriamente. ${ }^{20}$ En el caso de que los beneficios se concentren desigualmente en ciertos grupos de población de acuerdo a la prucba de Pareto, el beneficio poblacional agregado excederá los costos también agregados, produciéndose la possibilidad de una compensación; sin embargo dicha compensación, que podría realizarse vía impuesto y subsiclios o bien repercusión de costos en precios, no necesariamente será efectuada. La información sobre quién gana y quién perde y si la compensación se efectúa o no es diluida en una cantidad que representa un beneficio neto o una razón de efectividad. ${ }^{20}$

Cuando los riesgos sean asumidos por un sector de la sociedad, como en el caso del riesgo ocupacional de por ejemplo, los trabajadores de un tiradero de residuos tóxicos,se plantea la necesidad de sí efectuar dicha compensación paretiana en forma monetaria. Este hecho nos conduce a una controversia en el plano ético: 
¿es un daño a la salud compensable en forma monetaria? ¿quiénes aceptarán este pago por riesgo sino quienes tienen menores opciones laborales o, como veremos en el siguiente punto, quienes están más desinformados? ̨qué implicaciones tiene este arreglo con repecto a un derecho universal a la salud? Estas preguntas expresan nuevamente un límite de fondo a la utilización del ACB o ACE como base informativa suficiente para una toma de decisiones que reflejen la elección de la sociedad. Una recomendación frente a este límite es que los tomadores de decisiones den a la cuestión de los derechos un papel distintivo en sus deliberaciones, y no incorporen estas consideraciones en una mezcla homogénea de costos y beneficios. ${ }^{20}$

\subsubsection{Distribuición desigual de la riqueza y del conocimiento sobre riesgos en la población}

La utilización de la técnica WTP para la medición de intangibles se basa por una parte en que los individuos tienen la experiencia más sustentada y detallada de cómo una elección y sus resultados afectan su bienestar particular y por otra, en que la preferencia y la disponibilidad para pagar tienden a reflejar las ganancias y pérdidas reales en la calidad de las vidas individuales. ${ }^{20}$

Existen sin embargo, dos límites fundamentales al uso de valoraciones obtenidas a través de esta técnica para fines de evaluación económica, ya que ésta implica la comparación interpersonal de dichas valoraciones. Por una parte, la medida de esta preferencia estará influida por el conocimiento que el individuo tenga sobre el riesgo, bien o daño en cuestión; dicho conocimiento no es mismo para todos los individuos y grupos sociales, produciéndose entonces una menor valoración de costos y beneficios en la población desinformada;esto equivale a un sesgo sistemático en contra de estos individuos o grupos. Por otra parte, existe una disminuición de la utilidad marginal de dinero a medida que avanzamos clesde un nivel de pobreza hasta un nivel de holgura económica, por lo que la valoración de un bien intangible a través de WTP estará influenciada por la utilidad que el sujeto dé a su dinero.Un ACB implica la comparación entre una pérdida valorada en una cantidad $x$ por un individuo $A$ y una ganancia valorada en una cantidad y por cl individuo B.Si embargo la exactitud de esta comparación con fines de decisión conlleva problemas, ya que - como señala Railton - es sistemáticamente regresiva. ${ }^{20}$

Estos elementos deben ser tomados en considcración como límites a las valoraciones obtenidas en base a la técnica de WTP no sólo porque implica sesgos sistemáticos de medición,sino también porque las decisiones tomadas en base a dichas mediciones, tenderian a reproducir el patrón de desigualdad existente ${ }^{20}$ en tanto desestimarían los costos y los benefícios de los menos favorecidos socio-económicamente hablando

\subsubsection{Valoración de costos y beneficios en las generaciones futuras}

La evaluación económica en el área ambiental considera que los problemas ambientales y sus alternativas de manejo tiene trascendencia intergeneracional.Por lo tanto aplica tasas de descuento a aquellos costos y beneficios que se extienden en el tiempo.Al no conocer las preferencias de las generaciones futuras, calculamos la tasa de descuento en base a las preferencias de la generación actual.Ello significa evaluar el valor global de una política mediante la sustitución de las preferencias de una porción particular de la población afectada - los que viven en el presente - por las preferencias de toda la población - las generaciones futuras - en las que sin embargo recacrán las consecuencias de la política. ${ }^{20}$

Estas reflexiones sobre la aplicación de las evaluaciones económicas al área ambiental señalan que su capacidad para proveer información sobre el grado de afectación de la salud y el bienestar de la población es limitada. Ello nos llevaría a la conclusión preliminar de que estas técnicas si bien no son suficientes para la empresa que frecuentemente se les asigna, si son necesarias a fin de explicitar y sistematizar en la medida de lo posible la información cuantitativa y comparable sobre 
cada una de las alternativas de manejo ambiental. Ello contribuye a la claridad y objetividad con la que la sociedad clige la forma de enfrentar el reto de la contaminación. Dicha clarificación es cada vez más necesaria en la medida que diversos grupos de interés toman conciencia de la importancia de esta área en los planes de desarrollo económico nacionales y en la orientación de los electores y consumidores. Consideremos detenidamente estos aspectos propiamente políticos del tema.

\section{5 - La Dimensión Politica en la Toma de Decisiones Ambientales}

El sustrato político en las evaluaciones y en el proceso de toma de decisiones es frecuentemente negado por los propios evaluadores e incluso por los tomadores de decisiones, quienes esperan de una evaluación - incluída la económica - un parámetro totalmente objetivo que brinde una base racional e inobjetable a sus decisiones sin la influencia de los caprichos de la política. ${ }^{30}$

De acuerdo a esta perspectiva, la elección de alternativas de acción será racional y estará motivada por el cálculo consciente de las ventajas, basándose en un sistema explícito y consistente de valores ${ }^{25}$. El agente racional al tomar una decisión, seleccionará la alternativa cuya consecuencia valora más alto en términos de sus objetivos. Esta postura cuyo sustrato es la Teoría de la Elección Racional (Rational Choice)se ha desarrollado en el plano de la toma de decisiones de la empresa privada. Sin embargo su aplicación en el ámbito público enfrenta dificultades, primordialmente porque asume al tomador de decisiones - en este caso el gobierno - como entidad holística, es decir conforma un todo homogéneo e integral, una unidad con un solo propósito. ${ }^{1}$

Tomando en consideración la experiencia de procesos de toma de decisiones. particularmente las públicas - se ha desarollado la perspectiva del llamado Esquema Incrementalista. En ella las decisiones se componen de subdecisiones, las cuales son resueltas en forma intermitente y secuencial. Reconoce que las organizaciones no son cntidades holísticas, sino que comprenden a múltiples grupos de interés quienes poseen diferentes perspectivas sobre elecciones estralćgicas $y$ sus respectivas instrumentaciones ${ }^{17}$.

Desde esta perspectiva la evaluación misma se coloca en un contexto político ya que los programas a evaluar son a su vez politicos: tienen patrocinadores legislativos $y$ simpatizantes, carreras administrativas que dependen de ellos,y el apoyo del personal del programa, clientes y grupos de interés; en este contexto y por su importancia en el proceso de decisión, los reportes de evaluación inevitablemente toman una postura política aún cuando buscan ser objetivos ${ }^{30}$.

En este contexto resulta claro que las decisiones son también un resultado de un proceso de negociación entre grupos de interés y reflejan más la situación de fuerza de dichos grupos, que la alternativa óptima ${ }^{18}$.

Incorporar el ambiente político de la evaluación conduce aún al evaluador más optimista a dudar de la probabilidad de tener un impacto útil en operaciones y decisiones de programas $^{30}$. Sin embargo la postura de este escrito al abordar este tema no es la de descalificar la importancia de evaluaciones profesionales en el área ambiental.Por el contrario, postulamos que es el realizarlas en forma políticamente ingenua lo que conduce a su irrelevancia 30 .

En este punto sería conveniente preguntarnos qué tan maleables pueden ser las evaluaciones económicas a diversos intereses políticos. Supongamos que se busca evaluar un proyecto de Ley de Desmonte para frenar el proceso de dcforestación acelerada en un país. Supongamos que un grupo de silvicultores y agricultores de empresas privadas realiza una evaluación de las medidas regulatorias: entre los costos contabilizarían la posible disminuición de sus ganancias debido a los límites estabelecidos, el pago de los impuestos, la disminuición de su planta laboral, el impacto en otras industrias relacionadas con la producción maderera, así como el costo de la infraestructura de vigilancia y capcitación de impuestos forestales. Bajo estas 
consideraciones el beneficio neto de la medida sería prácticamente nulo. Supongamos ahora que los grupos de ciudadanos interesados en la preservación de la biosfera auspician la realización de otro estudio costo-beneficio de la misma ley: debido a su objetivo de protección, selecionarán un método que les permita estimar el valor intangible de la existencia de bosques, por ejemplo, la disponibilidad para pagar por parte de la población por la existencia de un parque nacional a través de cuotas de entrada; además estimarán costos más reducidos de instrumentación de la medida considerando la existencia previa de una infraestructura utilizable para otros fines de vigilancia y fiscalización simultáneos; también podrían, planteando límites espacio-temporales suficientemente amplios, ennumerar múltiples beneficios derivados de la conservación de los bosques, desde la preservación de las especies cuyo hábitat es la zona boscosa, el impedimento de alteraciones metcorológicas en cuanto a nivel pluviométrico y temperatura incluso fuera del territorio nacional, hasta el beneficio en la salud fisica y mental de la población derivado de la existencia y utilización de espacios estético-recreativos.

En una sociedad de valores e intereses diversificados que se manifiesta en el área ambiental - como en otras - pueden realizarse diversas evaluaciones económicas que propongan como óptima o racional aquella opción que beneficie en mayor medida sus intereses. Ello equivale a afirmar la posibilidad técnica de manipulación de estos instrumentos de análisis en base a los fines perseguidos y los supuestos bajo los cuales se realizan ${ }^{\text {is }}$. Mientras no exista un acuerdo en estos fines y los supuestos del análisis, la evaluación económica no legitima las decisiones en la materia ya que sólo indica la opción más adecuada al fin perseguido, y no la alternativa más racional entre una amplia variedad de objetivos.

Las evaluaciones económicas son manipuladas al encontrarse inmersas en un contexto crecientemente politizado como lo es el tema ambiental. En efecto, en torno a él se estan definiendo y creando nuevos grupos con nuevas demandas. La ciencia política ha concebido este surgimiento de los últimos años como un cambio en el eje que define el conflicto social y político de nuestro tiempo. Durante el siglo pasado y las primeras décadas del presente el factor proponderante del conflicto social se ubicaba clásicamente en la esfera de la propiedad de medios de producción y condiciones salariales. El advenimiento de la segunda mitad del presente siglo da lugar a un nucvo espacio de conflicto definido alrededor del control de las organizaciones y del saber tecnológico que lo posibilita ${ }^{8}$. El ejemplo más claro de este cambio es el surgimiento de los grupos ecologistas, quienes no demandan mejoras salariales o un control de los medios de producción, sino en primer lugar, información abicrta sobre la situación ambiental y en consecuencia, la intervención en la toma de decisiones que como ciudadanos y habitantes de un área les afectan, y en segundo lugar, la defensa de la identidad de su comunidad en base a nuevos valores:la protección del ambiente por sí mismo, y por sus relaciones con el bienestar de las generaciones presentes y futuras ${ }^{28}$. Dcbito a la dinámica organizativa de estos grupos, tanto como por cl creciente arraigo de estos valores en la ciudadanía, el tema ambiental no sólo revela un cambio cultural y social, sino propiamente politico, ya que se ha convertido incluso en un factor de peso en la definición de las preferencias clectorales.

Ahora bien, moldeabilidad de las argumentaciones en pro y en contra de diversas alternativas de acción a la problemática ambiental que subyace a la posibilidad de manipulación de las evaluaciones económicas tiene un límite: en la medida en la que la información y los valores de protección ambiental logren un arraigo en todos los sectores de la sociedad, se hará imperativa la toma de decisiones que no sólo satisfaga intereses de ciertos grupos, sino de la población en su conjunto, ya que la selección de medidas que no resuelvan la amenaza ambiental o bien que no distribuyan equitativamente los costos de dichas medidas, pueden poner en riesgo la estabilidad y la legitimidad gubernamental en largo plazo.

La sensibilidad para elucidar la amplia 
dimensión de la problemática ambiental corresponde tanto a los evaluadores que con su trabajo proporcionan un insumo fundamental para la definición de políticas al respecto,como a los grupos sociales activos en la materia y a los tomadores de decisiones mismos.

\section{6 - Conclusiones}

La evaluación económica es una herramienta que organiza y proveć una parte considerable de los insumos informativos para la toma de decisiones en materia ambiental. Debito a sus limitaciones para valorar información relativa al bienestar de la población, así como por su posibiliclad de ser manipulada en un contexto político de intereses diversificados, es preciso no considerarla como el único insumo necesario a la toma de decisiones en el área ambiental,sino como una ayuda importante en dicho proceso. Sus debilidades deben explicitarse a la vez que complementarse con otras formas de análisis.

Sus ventajas como herramienta de análiyis radican en explicar y organizar la información base para la toma de decisiones entre alternativas que se vuelven comparables a través de una razón costo-efectividad o un beneficio neto.Varios de los límites señalados en este artículo no constituyen obstáculos para su aplicación en el árca ambiental, sino retos a resolver como parte del perfeccionamiento de estas técnicas económicas. Presentamos aquí algunas propuestas que esperamos contribuyan a perfeccionar las formas de medición de la evaluación económica así como incorporar aspectos fundamentales de orden cualitativo en la información conducente a la toma de decisiones ambientales. Estas últimas sugerencias provienen de las recomendaciones generalmente aplicables a los estudios de evaluación con un alto contenido político.

1. Es preciso impulsar un desarollo metodológico para medir adecuadamente todos los costos y los beneficios de las distintas opciones de manejo ambiental, incluyendo los domésticos y personales.

2. Resulta un imperativo disminuir las diferencias informativas de la sociedad en cuanto a los riesgos ambientales, tanto una respuesta a las demandas de grupos cada vez más amplios en la sociedad, como porque a mayor información, se reduce el sesgo sistemático contra la población desinformada.

3. La información sobre la distribución de costos y beneficios en la población puede provecrse si los costos y bencficios del ACB se presenta en forma desagregada, es decir, a traves de una gráfica estrella en lugar de una sola recta: en esta gralica cada cje representaria a cada actor social en el que incide alguna proporción clel beneficio y del costo global de las medidas evaluadas. Esta desagregación resultaría particularmente pertinente para considerar la dimensión política de la toma de decisiones en materia ambiental.

4. Otras limitaciones señaladas, como el sesgo hacia los valores de la generación presente o las diferentes valoraciones sobre costos y beneficios por los niveles de utilidad marginal decrecicnte entre los distintos grupos de ingreso son por el momento inevitables. Sin embargo el primero es políticamente justificable en tanto los tomadores de decisiones son responsables ante los valores de su propia gencración. ${ }^{23}$ Lsta opción no sólo debe ser inevitable sino explícita.

5. Las lécnicas de evaluación económica deben ser crecientemente utilizadas no sólo para la toma de decisiones, sino para informar sobre éstas, debido a su capacidad sistematizadora de la información considerada. La sociedad puede y dcbe asumir la responsabilidad de las decisiones sobre recursos escasos aunque éstas planteen disyuntivas éticas crucles. Esa es la base de una decisión legítima.

6. Los dificrentes elementos referidos como condiciones de incertidumbre, tales como la relativa ignorancia sobre efectos acumulativos $y$ de largo plazo,involuctan un potencial catastrófico no cuantificado,y por lo tanto ausente de las evaluaciones económicas. No obstante no debe ser ignorado en el proceso de decisión.

7. El evaluador tendrá mejores resultados (a nível de utilización de su estudio) si es consciente de la dinámica polílica que subyace 
a su evaluación y explicita los presupuestos bajo los cuales esta evaluación es realizada.Por otra parte, la creciente politización del área ambiental implica una rápida difusión de los criterios de protección ambiental a nivel de ciudadanos, consumidores y electores, lo que pone un límite a la manipulación deliberada de la evaluación en favor de cicrtos grupos.

8. La vinculación entre evaluadores y tomadores de decisiones resulta clave a lin de que la evaluación económica, más que un ejercicio académico, constituya un apoyo pertinente para la toma de decisiones en materia ambiental.

Por último subrayamos que debido a la dinámica tecnológica, económica y la inherente a los valores culturales, así como al avance de la ciencia económica, la evalución cconómica no debe considerarse como un instrumento estático y definitivo, sino por el contrario como un proceso que permite su propio ajuste a la evolución en el tiempo de los factores que trata de medir y sistematizar.

GUTIÉRREZ ZÚNIIGA, C. et al. Avaliação econômica e tomada de decisão em saúcle ambiental. Rev.Saúcle Pública,28: 153-66, 1994. Devido à crescente deterioração ambiental $e$ as suas possiveis conseqüências para a saúcle da polulação,torna-se necessário priorizar o desenho e instrumentação de politicas que controlem as atividades econômicas, considerando a proteção à saúde humana e ao próprio ambiente. É preciso que essas politicas considerem a factibilidade econômica das alternativas de proteção existentes. Não obstante, pela quantidade de interesses na área ambiental, outros fatores como o social e o político também devem ser considerados. A avaliaçĩo econômica tem sido vista como fundamental para a tomada de decisões nessa matéria. Foi analisada a capacidade desta ferramenta para organizar de forma sistemática e comparável os custos e os beneficios de alternativas para a solução dos problemas ambientais.Foram sintetizadas as principais caracteristicas dos estudos de custo-bencficio e custo-efetividade, as formas de avaliação econômica do ambiente $e$ as particularidades desta área para a aplicação dessas técnicas de análise. Foram analisados os limites encontrados nessas ferramentas para calcular os custos não monetários dos riscos ambientais e dos danos consequentes à saúde, como a dor, o solirimento ou a incapacidade de pessoas economicamente inativas, sendo que estes aspectos constituem o desafio metodologico da avaliação econômica na área. Analisa-se a importância de ampliar a informação para a tomala de decisões a respcito da saúde ambiental em aspectos como a distribuiçĩo dos custos e os beneficios cntre os diferentes grupos sociais. Por último, assinala-se tanto a crescente politização do tema ambiental,como a possibilidade técnica de manipulação dessas ferramentas de análise.Partindo desses elementos, destaca-se a necessiclade, por parte dos avaliadores, de tomar a consciência das implicações políticas dos seus estudos,assim como da importância da sua relação com as pessoas que tomam decisões para alcançar pertinência no exercício acadênico.

Descritores: Análise custo-benefício. Tomada de decisões. Saúde ambiental, cconomia.

GUTIÍRRREZ-ZÚÑIG $\Lambda, C$. et al. [Economic evaluation and decision-making in environmental health]. Rev. Saúde Píblica,28:153.66, 1994. Due to the increasing deterioration of the enviroment and its possible consequences for the health of the population the design and implementation of policies for the control of economic activities according to criteria for the protection of human health and the environment itself have become an unquestionable need. Such policies would incluede the economic feasibility of existing alternatives for protection. Due to the huge interests involved, however, other factors, both social and political should also be taken into consideration. Economic evaluation has been seen as a promising founclation for the decision making process in this subject. This tool's capacity systematically to organize and compare the costs and the benefits of the alternatives for the solution of enviromental problems is analysed.The main chameteristics of cost-benefit and cost-eflectiveness studies are summarized, as well as the possible forms of economic evalution, of the enviroment and the detailsof this ficld for the application of such techiniques of analysis. The limits of these tools for the quantifycation of the non-monetary costs of environmental risks and the consequent damage to health, whethea as pain,suffering or the disability of non-conomic ally active persons are set out as these constitute the methodological challenge of the economic evaluation in the area. The importance of the cnhancement of information input for the decision-making process relating to environmental health issues such as the distributions of costs and bencfits among the different social groups is scrutinized. Lastly, the growuing political concern with environment issues is stated, as well as the techinical possibility of the manipulation of these amalytical tools. On the basis of these elements, the need for evaluators to be conscious on the political implications of their studies, as well as the importance of their relationship with the decision-makers in view of the need for effective relevance to current environmental issues

Keywords: Cost benefit analysis. Decision making. Environmental health, economy. 


\section{Referências Bibliográficas}

1. ALLISON, T. apud Pennings, J.M. ed. Decision making: an organizational behaviour approach. Princeton, Markus Wiencr Publ., 1986.

2. CHESNUT, \& ROWE, apud Margulis, S. Back-of-the-envelope estimates of environmental alamage costs in Mexico. Washington, World Bank, 1992. (Policy research WPS, 821).

3. CULLIS, J.G. \& WEST.P.A. The economics of health:an introduction. New York, Universily Press, 1979.

4. DRUMMOND, M.F. \& STODDART. G.L. Principles of economic evaluation of health programmes. World Health Stat.Q., 38:1-18, 1985.

5. ENVIRONMENTAL PROTECTION AGENCY. Regulatory impact analysis on the national ambient aiv quality standaits for particulate matter. Research Triangle Park, 1984.

6. FAHS, M. C. et al. apud Landrigan, P.J. Current status of occupational disease in the United States. J.Occup.Med., 1:97.100, 1992.

7. GHANA HEALTH ASSESSMENT PROJECT TEAM. A quantitative method of assessing the health impact of different diseases in less developed coutrie. Int. J. Epidemiol., 10:73-80, 1981.

8. HABERMAS, J. Crisis del capitalismo tardío y posibilidades de la democracia. Culad. Políticos, 19: 12, 1979.

9. HAENSZEL, W.A. Standarized rate for mortality defined in units of lost ycars of life. Am.J.Public Ileallh, 40:17-26, 1950

10. KRUPNICK, A.J.\& PORTNEY, P. R. Controlling urban air pollution: a benefit-cost assessment. Science, 252:522, 1991

11. LANDRIGAN, P.L. Curtent status of occupational disease in the united states. J. Occup. Med., 1:97-100, 1992.

12. MARGULIS, S. Back-of-the-envelope estimates of environmental damage costs in Mexico. Washington. World Bank, 1992. (Policy Research WPS, 824).

13. MELNICK, S. R. The politics of benefit-cost analysis. In: Hammond, B. \& Coppock, R. ed. Valuing health risks, costs, and benefits for environmental decision making. Washington, D.C., National Academy Press, 1990. p. 20-2

14. MEXICO. Ley Federal de Metrologia y Normalización, expedida el $1^{2}$ de julio de 1992.

15. MILLS, A. \& GILSON, L. Reestructuring the health sector in developing contries. London, School of Hygiene and Tropical Medicine, 1991.

16. PEARCE,W.D. Economía ambiental. México, Fondo de Cultura Económica, 1985.

17. PENNINGS., J. Decision making macro-organizational behavior. In: Pennings, J., ed. Decision making: an organizational behaviour approach.Princeton, Markus Wiener Publ, 1986. p. 197-8.

18. PENNINGS, J. Decision making: organizational strategy. In: Pennings, J., ed. Decision making: an organizational behaviour approach. Princeton, Markus Wiener Publ., 1986 .p. 109-10.
19. PRUD'IIOMME, R. Le ménagement de la nature:des politiques contre la pollution. Paris, Dunod, 1980.

20. RAILTON, P. Benefit cost analysis as a source of information about wellare. In: I Iammond, B.\& Coppock, R., ed. Valuing heallh risks, costs, and benefits for environmental decision making. Washington, D.C., National Academy Press, 1990 .p.73

21. REPETTO, R. Accounting for environmental essets. Sci.Am., 266:64-74, 1992.

22. ROSENFIELD, P.L. The management of schistosomiasis. Washington, D. C., Resources for the Future, 1979.

23. RUSSELL, $M$. The making of cruel choices. In:Hammond, B.\& Coppock, R., ed. Valuing health risks, costs and benefits for environmental decision making. Washington, D. C., National Academy Press, 1990. p. 20-2

24. ROBEIRTS, M.J.Logical and philosophical problems of allocating scarce health care resoucers. In:Takemi Simposium on International IIealth, 12, Boston, s. d. p.61

25. SCIIELLING, T.apud Allison, G.T.Conceptual models and the Cuban missile crisis.In:Pennings, J., ed. Decision making: an ovganizational behaviour approach.Princeton, Markus Wiener Publ., 1986. p. 315.

26. SUAREz, R .Módulo de análisis de costo efectividad aplicado a los seivictos de salual: análisis de costas clemancla y planificación de servicios de salud. Washington, D.C., OPS, 1990

27. TIIOMPSON, M.S. Willingness to pay and accept risks to cure chronic disease. Am.J.Public Ilealth, 76:392-6, 1986

28. TOURAINE, A.Reacciones antinucleares o movimiento antinuclear. Rev.Mexicana Sociol, 44:696, 1982.

29. VANIOONGEREN, J. et al. Integrated environmental and economic accounting: a case study for Mexico. Washington, The World Bank, 1991. (Environment Working Paper $n^{2} 50$ ).

30. VENEY, J. E. \& KALUZNY, A.D. Evaluation and decision making for health services. Michigan, Health Administration Press, 1991.

31. WARNER, K.E. \& LUCE, B.R. Introduction to cost benefit and cost cffectiveness analysis. In: Cost-benefit and cost-e/fectiveness analysis in health care:Principles, practice and potential.Ann Arbor, Health Administration Press, 1982.

Recebido para publicação em 1.10 .1993 Aprovado para publicação em 8.3.1994 